\title{
Reduced Fasting Protocol for Endoscopic Percutaneous Gastrostomy in Intubated Patients
}

\author{
Catherine Ho, John Culhane
}

Department of Surgery, Arrowhead Regional Medical Center, Colton, USA

Email: CulhaneJ@armc.sbcounty.gov

Received May $1^{\text {st }}, 2013$; revised June $13^{\text {th }}, 2013$; accepted June $28^{\text {th }}, 2013$

Copyright (C) 2013 Catherine Ho, John Culhane. This is an open access article distributed under the Creative Commons Attribution License, which permits unrestricted use, distribution, and reproduction in any medium, provided the original work is properly cited.

\begin{abstract}
Background: Previous studies have shown that ICU patients receive only a fraction of their calculated nutritional goals, and that cumulative caloric deficit in the ICU has been correlated with poor outcome. One reason for this underfeeding is the frequent interruption of enteral nutrition. Many ICU patients receive enteral feeding formula via a nasogastric (NG) tube. Feeding is typically held for several hours prior to procedures due to the theoretical risk of aspiration. An alternative is to continue feeding up until the procedure begins, then stop the feeding and place the NG to suction. This evacuates the contents of the stomach and minimizes the risk of aspiration, while reducing the interruption of feeding that can result in malnutrition. Methods: This study is a review of prospectively gathered data including 55 sequential patients who underwent bedside percutaneous endoscopic gastrostomy (PEG) placement in a mixed ICU under a reduced fasting protocol. This was compared with a historical cohort of 33 critically ill trauma patients who fasted for at least 8 hours prior to the procedure. Under the reduced fasting protocol, enteral feeding via NG was continued up until the time of the procedure. The NG was then placed to suction, and sedation was given. The NG was left in place until the esophagus was cannulated, then it was removed. The PEG was placed in standard fashion, and feeding was resumed via the PEG immediately following the procedure. Results: We have documented no peri-procedural vomiting or aspiration. New diagnosis of pneumonia within 3 days occurred in 2/55 of the feeding group and 4/33 of the fasting group. Overall mortality was higher for the feeding group: $13 / 55$ vs. 1/33, however when adjusted for baseline characteristics, the difference was no longer significant. Under our protocol, the interruption of feeding averaged approximately one hour, rather than the 8 or 9 hour interruption required by standard protocols. This saved an average of approximately 700 kilocalories per patient. Conclusion: We believe that continuing feeding via NG up until the time of bedside PEG is safe and advantageous for intubated patients in the ICU. Since there was minimal gastric content, it may also be safe for other procedures.
\end{abstract}

Keywords: Aspiration; Gastrostomy; PEG; NPO; Pneumonia; ICU; Feeding; Enteral; NG Tube; Fasting

\section{Introduction}

Adequate nutrition is essential for recovery from severe illness. ICU patients typically receive only a fraction of their calculated nutritional goals [1], and cumulative caloric deficit in the ICU has been correlated with poor outcome $[2,3]$. One reason for this underfeeding is the frequent interruption of enteral nutrition. Many ICU patients receive enteral feeding formula via a nasogastric or orogastric tube. Tube feeding is typically held for several hours prior to procedures due to the theoretical risk of aspiration.

The American Society of Anesthesiologists has re- cently published practice guidelines for recommended fasting intervals for elective surgery in healthy patients [4]. These guidelines are often applied to intubated patients receiving enteral feeding as well, but they are not intended for this patient population. There is little evidence in the literature to guide decisions about pre-procedural fasting in intubated patients with feeding tubes.

In an effort to minimize interruptions in feeding, we have developed a protocol to minimize the NPO period for bedside PEG. We continue feeding up until the procedure begins, then stop the feeding and place the NG tube to suction. This evacuates the stomach and minimizes the risk of aspiration. This protocol has the benefit 
of reducing interruptions of feeding that can result in malnutrition.

\section{Methods}

This study is an analysis of prospectively gathered data. Fifty five sequential patients underwent bedside PEG placement in the ICU according to our protocol. All patients were already intubated. Enteral feeding via NG was continued up until the equipment was mobilized for the procedure. At that time, the NG was placed to suction, and sedation was given. The NG was left in place until the esophagus was cannulated, then it was removed. The PEG was then placed in standard fashion, and feeding was resumed via the PEG immediately after the procedure. These patients compose the feeding group.

We used our trauma registry to identify a control group of patients who had PEG's placed after the standard NPO period, usually after midnight the previous day. These patients compose the fasting group.

A qualitative description of the stomach contents and any evidence of aspiration during the procedure were documented when the procedure was done. The charts were reviewed to determine whether pneumonia was diagnosed within three days of the procedure. Radiology records were reviewed to assess any radiologic evidence of new pneumonia during this time period.

The procedures for the feeding group were all performed by the second author, a surgeon and intensivist, with assistance from residents. The procedures for the fasting group were mostly performed by the gastroenterology group covering our hospital. A few of the patients who had PEG's done by the surgeon were fasting for other reasons and were included in the fasting group. Diagnosis of pneumonia was made using American Thoracic Society guidelines: new infiltrate, fever, purulent sputum, and leukocytosis.

The study was approved by the Arrowhead Institutional Review Board.

\section{Patients}

The patient population was a group of critically ill patients at Arrowhead Regional Medical Center, a Level 2 Trauma Center located in Colton, California. The population was a mixture of medical and surgical ICU patients, including many trauma patients.

\section{Statistics}

Comparisons between groups were made using Student's t-test for continuous data and Fisher's Exact Test or Pearson's Chi square test for categorical data. Microsoft Excel spreadsheet was used to tabulate the data and to perform the calculations.

\section{Results}

\subsection{Baseline Characteristics}

\begin{tabular}{cccc}
\hline & Feeding & \multicolumn{2}{c}{ Fasting } \\
\hline Age & 47.7 & 45.8 & $\mathrm{p}=0.6$ \\
Gender (m/f) & $36 / 19$ & $20 / 13$ & $\mathrm{p}=0.7$ \\
Trauma & $22 / 55$ & $33 / 33$ & $\mathrm{p} \leq 0.001$ \\
$\begin{array}{c}\text { Antibiotics } \\
\text { (For PNA) }\end{array}$ & $33 / 55$ & $19 / 33$ & $\mathrm{p}=0.7$ \\
\hline
\end{tabular}

\subsection{Outcome}

\begin{tabular}{cccc}
\hline & Feeding & \multicolumn{2}{c}{ Fasting } \\
\hline Pneumonia & $2 / 55$ & $4 / 33$ & $\mathrm{p}=0.2$ \\
X-ray change & $6 / 55$ & $4 / 33$ & $\mathrm{p}=1$ \\
$\begin{array}{c}\text { Observed } \\
\text { Aspiration }\end{array}$ & $0 / 55$ & $\mathrm{NR}$ & \\
Mortality & $13 / 55$ & $1 / 33$ & $\mathrm{p}=0.004$ \\
Trauma Mortality & $2 / 22$ & $1 / 33$ & $\mathrm{p}=0.6$ \\
\hline
\end{tabular}

\subsection{Description of Gastric Contents}

\begin{tabular}{cc}
\hline Trace fluid & 9 patients \\
Coating of formula on mucosa & 9 patients \\
Clean & 40 patients \\
\hline
\end{tabular}

We have documented no peri-procedural vomiting or aspiration. In addition, when the stomach was visualized during endoscopy for PEG, the gastric contents have been minimal. There was no large pool of gastric contents in any patient. We used a qualitative assessment of the gastric contents because it would have been difficult to measure the exact amount. Residual gastric contents are often viscous due to thick formula or mucous secretions. Aspirating all of the fluid to measure the volume would have added significant time to the procedure, possibly increasing risk to the patient.

The overall mortality for the feeding group is higher, however we believe this is due to baseline differences. The records for the fasting group were obtained from a trauma registry; hence they are all trauma patients. The feeding group represents a mixed ICU population. Mortality in the trauma subgroup of the feeding group was not significantly higher than that of the fasting group. Trauma patients tend to be healthier at baseline, therefore those who survive their initial injuries long enough to need a PEG are more likely to survive to discharge.

In order to investigate whether the mortality could be related to the feeding protocol, we examined the cause of 
death for every patient in the feeding group who died. The results were:

Care withdrawn due to brain disease or injury: 10;

Tracheo-innominate fistula: 1 ;

Abdominal sepsis due to necrotic large bowel on high dose steroid therapy: 1 ;

Pneumonia 5 months after the PEG was placed: 1.

Almost all of the deaths were a result of family and physicians withdrawing life support due to poor neurologic prognosis. Only one death was due to pneumonia, and this happened 5 months after the PEG procedure. It is unlikely that any of these deaths were due to the feeding protocol.

Although there was no gross vomiting or aspiration during the endoscopy, there could have been micro aspiration, which theoretically could have led to pneumonia. To investigate this possibility, we looked back to see which patients had treatment for pneumonia initiated within three days following the procedure. We also looked to see if there were any changes in chest X-rays following the PEG. There was no evidence of increased pneumonia or chest $\mathrm{x}$-ray changes for the feeding group.

Under our protocol, the interruption of feeding averaged approximately one hour, rather than the 8 or 9 hour interruption required by standard protocols. This saved an average of about 700 kilocalories per patient. This does not take into account multiple periods of fasting due to cancellation and rescheduling of procedures.

\section{Discussion}

There is little evidence of any improvement in outcome due to pre-procedure fasting in intubated ICU patients, but there is evidence that underfeeding may be harmful. Multiple studies have shown a statistically significant reduction in mortality and infectious complications with early enteral nutrition $[2,5,6]$. The initiation of enteral nutrition leads to improved patient outcomes as it may reduce inflammatory cytokines, improve immune function, reduce septic complications, and promote wound healing. Overall, studies have demonstrated that enteral feeding reduces infectious morbidity, hospital length of stay, and multiple organ failure [6]. Critical care nutrition guidelines therefore support early enteral nutrition as the preferred method of providing nutrition to critically ill patients $[2,6,7]$.

Critically ill patients generally exhibit increased metabolic rate, however, their high metabolic needs are often inadequately met. Observational studies have shown that up to $40 \%$ of critically ill patients receive no nutritional support during their ICU stay. Also, $60 \%$ of patients who stay in the ICU at least 3 days remain unfed for 48 hours or longer [6]. In those patients who are receiving nutriation, interruptions in feeding occur when procedures ei- ther at the bedside or in the operating room require a fasting interval prior to the intervention. Underfeeding in surgical or mixed medical-surgical ICUs is a widely recognized issue [1].

There is no consensus on withholding feeding for procedures. Schneider at al. performed a survey study comparing the practices of anesthesia, anesthesia critical care, surgical critical care, and medical critical care departments in the length of time necessary for withholding enteral feeding from intubated patients before scheduled procedures, including extubation and tracheostomy [8]. Significant findings of this study showed that for scheduled tracheostomy, surgical critical care departments reported withholding enteral feeding for a median of 4 hours versus all other departments with a median of 6 hours. The fasting interval prior to a procedure in an intubated patient is still debatable and varies in practice.

The American Society of Anesthesiologists has published recent practice guidelines for recommended fasting intervals for elective surgery in healthy patients [4]. These guidelines are often applied to critically ill patients receiving enteral feeding, although they were not intended for this population. The standard 8-hour fasting period is often extended when procedures are delayed from the scheduled time. The ideal caloric intake for most patients in critical care is 25 to $30 \mathrm{kcal} / \mathrm{kg} / 24$ hours. During the 8-hour fasting interval, an average loss of approximately 700 kilocalories occurs, thereby increasing the cumulative caloric deficit.

The preoperative standard fasting interval was established due to concerns for aspiration of gastric contents; however the risk posed by gastric contents may be overstated. Previous studies have examined the utility of the use of gastric residual volumes (GRV) in the monitoring of tube-feeding intolerance. The frequency of gastric residual volume assessment and the threshold at which feeding should be withheld is controversial. There are various feeding protocols and recommendations reported in the literature, mostly with a threshold GRV of $</=200$ $\mathrm{ml}$. A recent study has shown that over a wide range of GRV, there is no correlation of GRV to aspiration events [9]. Sorosky et al. developed a protocol which initiates a full caloric target delivery rate, allows a larger GRV threshold of up to $500 \mathrm{ml}$ before withholding feeding, and decreases the number of GRV assessments to once per day [10]. The study showed no correlation between GRV values and the rate of pneumonia or other outcome variables such as the length of ICU stay, duration of mechanical ventilation, or ICU and hospital mortality. In the subgroup of patients who developed ventilator-associated pneumonia, patients with and without VAP had similar GRV's during the study. Of the vomit/regurgitation episodes in 13 patients only one patient developed ventilator associated pneumonia (VAP). In the studies of the bene- 
fit of early enteral feeding, there was a small statistically significant increase in rates of vomiting; however, no complications resulted from the emesis [11]. GRV has also been evaluated in comparing gastric to postpyloric feeding. The use of postpyloric feeding showed reduced GRV but no difference in aspiration pneumonia was seen between the two routes of feeding [7].

There are theoretical reasons why intubated patients may not benefit from pre-procedural fasting. Fasting does not guarantee an empty stomach. Gastric contents contain a mixture of feedings, gastric and salivary secretions and duodenal reflux. Endogenous secretions can amount to as much as 4.5 liters per day [12]. Even when the patient is NPO we rely on gastric peristalsis and aspiration via gastric tubes to ensure gastric emptying. Regular checks for residual volume can prevent accumulation of large gastric volumes even during continuous tube feeding. A cuffed endotracheal tube may provide some protection against aspiration, though it is not absolute. Suction through the NG tube can evacuate residual stomach contents immediately prior to the procedure.

Previous studies have shown that the fasting period for procedures in intubated patients can be safely reduced or eliminated. In a study from Shriners Burn Institute, Jenkins et al. studied the safety and efficacy of providing enteral support throughout the operative procedures in eighty burn patients [13]. Forty patients received enteral feeding throughout 161 surgical procedures, and $40 \mathrm{pa}-$ tients had enteral support withheld during 120 procedures. Nutritional status, calorie counts, and infectious complications were studied during the first 4 weeks after the burn injury. The unfed group was found to have significant caloric deficit, increased occurrence of wound infections, and increased albumin supplementation. No patients in either group experienced aspiration. This study supports the use of peri-operative enteral feeding to maximize nutrition for better outcomes and does not show a negative impact of peri-operative feeding such as aspiration.

Pousman et al. implemented a new protocol to reduced fasting interval in mechanically ventilated trauma patients undergoing selected operative and non-operative procedures [14]. The standard group adhered to the minimum 8-hour fast prior to procedures while the intervenetion group permitted patients to receive small bowel feeding up until the time of the procedure. Intragastric feeding continued until 45 minutes prior to the procedure, at which time the gastric tube was flushed and aspirated. Although no statistical significance was shown, there was a trend demonstrating increased delivery of enteral nutrition and faster achievement of nutrition goals in the intervention group. Furthermore, there were no differences in rates of infectious complications including ventilator-associated pneumonia between the two groups.
The authors thus concluded that a reduced fasting protocol was possible for selected operative procedures with improved nutrition support and no increase in complications.

Silent aspiration is common, but generally clinically insignificant [15], however, witnessed aspiration during intubation that leads to pneumonia is rare, even in patients who have not been fasting. Thibodeau et al. recorded a 3.5\% rate of aspiration during ER intubations, with none of these patients developing pneumonia [16]. It would be difficult to show that any intervention leads to an outcome difference for such a rare event. The evidence for benefit of fasting often relies on the surrogate variable on volume of gastric contents [4]. A strength of our study is that gastric contents which could potentially cause aspiration were visualized directly. Gastric volume was found to be minimal using our protocol.

This study is subject to certain limitations. The NPO group was a historical control. Information was not prospectively gathered as it was for the feeding group. Aspiration during the procedure for the NPO population was not specifically mentioned, but no such event was ever documented on review of the procedure reports. The gastric contents were seldom described. Many of our patients were receiving empiric treatment for pneumonia at the time of the PEG procedure. The incidence is consistent with previous reports in the literature. Two recent studies of ICU enteral feeding practices reported ventilator associated pneumonia incidence of $41 \%$ to $50 \%$ [14, 17].

\section{Conclusion}

In this study, we have shown that it is safe to eliminate a fasting interval prior to percutaneous endoscopic gastrostomy in intubated patients receiving enteral feeding. Eliminating the fasting period does not lead to increased complications from aspiration. Our protocol served to minimize interruption of enteral feeding, thereby maximizing caloric intake of critically ill patients. We believe that continuing enteral feeding via NG up until the time of bedside PEG is safe and advantageous for intubated patients in the ICU. In addition, since we never saw more than minimal gastric contents for any patient, the risk of vomiting and aspiration should be low for any procedure. This could have implications for the time needed to hold feeding for other procedures, including surgery in the operating room.

\section{REFERENCES}

[1] M. E. Hise, K. Halterman, B. J. Gajewski, M. Parkhurst, M. Moncure and J. C. Brown, "Feeding Practices of Severely Ill Intensive Care Unit Patients: An Evaluation of Energy Sources and Clinical Outcomes," Journal of the 
American Dietetic Association, Vol. 107, No. 3, 2007, pp. 458-465. doi:10.1016/j.jada.2006.12.012

[2] S. Villet, R. L. Chiolero, M. D. Bollmann, J. P. Revelly, R. N. Cayeus, J. Delarue and M. M. Berger, "Negative Impact of Hypocaloric Feeding and Energy Balance on Clinical Outcome in ICU Patients," Clinical Nutrition, Vol. 24, No. 4, 2005, pp. 502-509. doi:10.1016/i.clnu.2005.03.006

[3] C. Faisy, M. Candela Llerena, M. Savalle, J. L. Mainardi and J. Y. Fagon, "Early ICU Energy Deficit is a Risk Factor for Staphylococcus Aureus Ventilator Associated Pneumonia," Chest, Vol. 140, No. 5, 2011, pp. 1254-1260. doi:10.1378/chest.11-1499

[4] American Society of Anesthesiologists Committee, "Practice Guidelines for Preoperative Fasting and the Use of Pharmacologic Agents to Reduce the Risk of Pulmonary Aspiration: Application to Healthy Patients Undergoing Elective Procedures. An Updated Report by the American Society of Anesthesiologists Committee on Standards and Practice Parameters," Anesthesiology, Vol. 114, No. 3, 2011, pp. 495-511. doi:10.1097/ALN.0b013e3181fcbfd9

[5] R. Dhaliwal and D. K. Heyland, "Nutrition and Infection in the Intensive Care Unit: What Does the Evidence Show?" Current Opinion in Critical Care, Vol. 11, No. 5, 2005, pp. 461-467.

[6] C. S. Doig, P. T. Heighes, F. Simpson, Sweetman and E. A. R. Davis, "Early Enteral Nutrition, Provided within 24 $\mathrm{h}$ of Injury of Intensive Care Unit Admission, Significantly Reduces Mortality in Critically Ill Patients: A Meta-Analysis of Randomized Controlled Trials," Intensive Care Medicine, Vol. 35, No. 12, 2009, pp. 20182027. doi:10.1007/s00134-009-1664-4

[7] D. K. Heyland, R. Dhaliwal, J. W. Drover, L. Gramlich and P. Dodek, "Canadian Critical Care Clinical Practice Guidelines Committee. Canadian Clinical Practice Guidelines for Nutritional Support in Mechanically Ventilated Critically Ill Adult Patients," Journal of Parenteral and Enteral Nutrition, Vol. 27, No. 5, 2003, pp. 355-373. doi: $10.1177 / 0148607103027005355$

[8] J. A. Schneider, Y. J. Lee, W. R. Grubb, J. Denny and C. Hunter, "Institutional Practices of Withholding Enteral Feeding from Intubated Patients," Critical Care Medicine, Vol. 37, No. 7, 2009, pp. 2299-2302. doi:10.1097/CCM.0b013e3181a007eb

[9] K. K. Kattelmann, M. Hise, M. Russell, P. Charney, M. Stokes and C. Compher, "Preliminary Evidence for a
Medical Nutrition Therapy Protocol: Enteral Feedings for Critically Ill Patients," Journal of the American Dietetic Association, Vol. 106, No. 8, 2006, pp. 1226-1241. doi:10.1016/i.jada.2006.05.320

[10] A. Sorosky, J. Lorber, E. Klinowski, E. Illgayev, A. Mizrachi, A. Miller, T. M. B. Yehuda and Y. Leonov, "A Simplified Approach to the Management of Gastric Residual Volumes in Critical Ill Mechanically Ventilated patients: A Pilot Prospective Cohort Study," Israel Medical Association Journal, Vol. 12, 2010, pp. 543-548. doi:10.1016/j.suc.2006.09.002

[11] J. B. Ochoa and D. Caba, "Advances in Surgical Nutrition," Surgical Clinics of North America, Vol. 86, No. 6, 2006, pp. 1483-1493.

[12] M. H. Delegge, "Managing Gastric Residual Volumes in the Critically Ill Patient: An Update," Current Opinion in Clinical Nutrition \& Metabolic Care, Vol. 14, No. 2, 2011, pp. 193-196. doi:10.1097/MCO.0b013e328341ede7

[13] M. E. Jenkins, M. M. Gottschlich and G. D. Warden, "Enteral Feeding during Operative Procedure in Thermal Injuries," Journal of Burn Care \& Research, Vol. 15, No. 2, 1994, pp. 199-205. doi:10.1097/00004630-199403000-00019

[14] R. M. Pousman, C. Pepper, P. Pandharipande, G. D. Ayers, B. Mills, J. Diaz, B. Collier, R. Miller and G. Jensen, "Feasibility of Implementing a Reduced Fasting Protocol for Critically Ill Trauma Patients Undergoing Operative and Nonoperative Procedures," Journal of Parenteral and Enteral Nutrition, Vol. 33, No. 2, 2009, pp. 176-180. doi:10.1177/0148607108327527

[15] E. J. Huxley, J. Viroslav, W. R. Gray, et al., "Pharyngeal Aspiration in Normal Adults and Patients with Depressed Consciousness," American Journal of Medicine, Vol. 64 , No. 4, 1978, pp. 564-568. doi:10.1016/0002-9343(78)90574-0

[16] L. G. Thibodeau, V. P. Verdile and J. M. Bartfield, "Incidence of Aspiration after Urgent Intubation," American Journal of Emergency Medicine, Vol. 15, No. 6, 1997, pp. 562-565. doi:10.1016/S0735-6757(97)90157-1

[17] J. B. A. MacLeod, J. Lefton, D. Houghton, C. Roland, J. Doherty, S. M. Cohn and E. S. Barquist, "Prospective Randomized Control Trial of Intermittent Versus Continuous Gastric Feeds for Critically Ill Trauma Patients," Journal of Trauma, Vol. 63, No. 1, 2007, pp. 57-61. doi:10.1097/01.ta.0000249294.58703.11 